\title{
Videojuegos y sociedades de control: la formación del cognitariado en las Global Game Jams
}

Video Games and Societies of Control: The Formation of Cognitariat in the Global Game Jams

Jeux vidéo et sociétés de contrôle: formation du cognitariat à la Global Game Jams

Luis Sebastián Ramón Rossi

\section{OpenEdition}

\section{Journals}

Edición electrónica

URL: http://journals.openedition.org/rccs/8581

DOI: $10.4000 /$ rccs.8581

ISSN: 2182-7435

Editor

Centro de Estudos Sociais da Universidade de Coimbra

Edición impresa

Fecha de publicación: 1 mayo 2019

Paginación: 101-124

ISSN: 0254-1106

\section{Referencia electrónica}

Luis Sebastián Ramón Rossi, «Videojuegos y sociedades de control: la formación del cognitariado en las Global Game Jams », Revista Crítica de Ciências Sociais [En línea], 118 | 2019, Publicado el 26 abril 2019, consultado el 21 marzo 2020. URL : http://journals.openedition.org/rccs/8581 ; DOI : https:// doi.org/10.4000/rccs.8581 


\section{LUIS SEBASTIÁN RAMÓN ROSSI}

\section{Videojuegos y sociedades de control: la formación del cognitariado en las Global Game Jams}

En este artículo tomamos la división deleuzoguattariana entre sujeción social (assujettissement) y esclavitud maquínica (asservissement machinique) para comprender la formación del cognitariado en las jornadas de desarrollo de videojuegos Global Game Jams. Después de contextualizar dichas sesiones y su importancia para la industria del desarrollo de videojuegos trabajaremos, por un lado, las dimensiones de la sujeción usuario-productor (apelación al emprendimiento, formación permanente, gestación de comunidades) y, por otro lado, las dimensiones de la esclavitud maquínica (dinámicas de trabajo flexible, fusión de juego y trabajo, división de tareas y proyectos, etc.).

Palabras clave: capitalismo; cognitariado; Global Game Jams; relaciones sociales; trabajo; videojuegos.

Palavras-chave: capitalismo; cognitariado; Global Game Jams; relações sociais; trabalho; videojogos.

\section{Introducción}

De acuerdo con Gilles Deleuze y Félix Guattari (1997), con el paso de las sociedades disciplinarias a las sociedades de control también han mutado las relaciones de poder, las formaciones de saber, los regímenes de signos y los modos de subjetivación. En particular, los filósofos franceses, inspirados por Mumford, señalan que en el Capitalismo Mundial Integrado (CMI) a la sujeción social disciplinaria (assujettissement), sustentada en la dualidad usuario-productor (asalariado-consumidor), se le ha sumado un nuevo modo de subjetivación ${ }^{1}$ que denominan esclavitud maquínica (asservissement machinique).

\footnotetext{
${ }^{1}$ Optamos por señalar que asservissement machinique es un modo de subjetivación dado que en el arco argumental deleuzoguattariano (que por su extensión sería imposible desarrollar aquí) esta modalidad no solo incorpora elementos de desubjetivación, como sugiere Lazzarato, sino que también, como señala Genosko, implica un tránsito hacia lo que Guattari denominaba una subjetividad maquínica de las sociedades posmediáticas. Esta última figura está implícita en las raíces de las líneas de fuga que abordaremos hacia el final de este escrito.
} 
Este segundo modo de subjetivación no encierra una connotación peyorativa, sino que se refiere a los sistemas hombres-máquinas a través de los cuales comunicación e información se efectúan en multiplicidades de elementos heterogéneos o agenciamientos ${ }^{2}$ de las formaciones del control (Deleuze, 1996; Rossi, 2018).

La complementariedad de ambos regímenes de subjetivación ha sido vista, por pensadores del posoperaismo italiano, como fundamental en la formación del cognitariado. En concreto, Franco Berardi (2009) ha subrayado que el capitalismo es la movilización de un pathos, o que el alma es puesta a trabajar, mientras que el proceso de trabajo cognitivo involucra transformaciones donde la comunicación y la imaginación se convierten en herramientas creativas primarias de producción de valor, y superficies de una esclavitud maquínica generalizada. Esta generalización, como señala la teoría deleuzoguattariana, implica que con la axiomática capitalista la valorización del capital se extiende a todo el cuerpo social, acompañada por una creciente importancia del capital constante sobre el capital variable (Hardt y Negri, 2002) y por una captura de múltiples semióticas por parte de la industria del ocio y de los agenciamientos massmediáticos. Para Lazzarato (2014) se trata de una radicalización de la postura marxista, en un tiempo en el que el maquinismo ha invadido completamente nuestra vida cotidiana constituyendo un capital social constante.

Apoyados en investigaciones más extensas, ${ }^{3}$ sostenemos que los videojuegos pueden ser pensados como agenciamientos específicos de las sociedades de control o controlatorios. En particular, las modalidades de desarrollo e invención que ellos implican ejemplifican la complementariedad entre sujeción social y esclavitud maquínica en la formación del cognitariado. Por ello, en las siguientes páginas proponemos un abordaje del desarrollo de videojuegos en las jornadas denominadas Global Game Jams (GGJ), con sede en la ciudad de Santa Fe (Argentina). Ensayamos así un doble aporte: por un lado, buscamos generar conocimiento sobre este tipo de jornadas, casi inexploradas en la literatura crítica, y, por otro, intentamos acrecentar la base empírica de las hipótesis derivadas de la teoría social deleuzoguattariana.

En las citadas jornadas de desarrollo tiene asiento una parte importante de la formación del cognitariado para la industria de videojuegos. En este

\footnotetext{
${ }^{2}$ No podemos desarrollar aquí la teoría del agenciamiento o de las componendas (traducción de agencement sugerida por Pardo). Para nuestro objetivo inmediato nos basta señalar con Deleuze que todo agenciamiento supone, ontogenéticamente, una multiplicidad de elementos heterogéneos atravesados por dos ejes (codificación y territorialización) y por cuatro coordenadas: fugas, territorios, estados de cosas y enunciados (Rossi, 2018).

${ }^{3}$ Este artículo contiene algunos ejes trabajados por el autor en su tesis doctoral "Videojuegos: agenciamiento y producción de subjetividad en las sociedades contemporáneas”.
} 
sentido, pedimos al lector que imagine un péndulo de movimiento constante con dos vectores, uno hacia la sujeción y el otro hacia la esclavitud maquínica. Siguiendo a Deleuze (1996), esa oscilación nos permitirá trabajar tres dimensiones íntimamente ligadas. Primero veremos funcionar al capital como punto de subjetivación bajo el sistema usuario-productor (Deleuze y Guattari, 1997), a través de regímenes significantes en los que quedan establecidas las figuras más recientes de la sujeción (empresario de sí, formación permanente, gestación de comunidades) (III). En una segunda fase comprenderemos las dinámicas del trabajo flexible y digital como espacios y tiempos, que se corresponden con un hombre que se ha vuelto ondulatorio bajo la fusión entre juego y trabajo, propia de la esclavitud maquínica (IV). Por último, abordaremos el proceso de trabajo por proyectos, la estructura de la división de tareas y la utilización de herramientas de desarrollo en un capitalismo abocado al producto y al prototipado (V). No obstante, antes de indagar dichas dimensiones, en los acápites siguientes contextualizaremos las jornadas GGJ y su importancia en la industria del desarrollo de videojuegos (I), al tiempo que señalaremos algunas estrategias metodológicas de nuestro trabajo (II). Finalmente, nos detendremos en dos ejes para seguir pensando acerca de estos problemas (modos de semiotización y desterritorializaciones). En concreto, los procesos de devenir que señalaremos bajo el segundo eje implicarán que las GGJ puedan ser consideradas como espacios de experimentación, cuya potencialidad permite atisbar una salida a la dinámica de la axiomática capitalista.

\section{Del desarrollo de videojuegos a las Global Game Jams}

La industria de videojuegos ha sido vista como un caso paradigmático del CMI, caracterizándose por métodos de acumulación basados en derechos de propiedad, en la contratación de trabajo global, la venta a escala mundial y la explotación cognitiva (Kerr, 2006; Dyer y De Peuter, 2009). Pero también, para Dyer (2015), el desarrollo de videojuegos ha forjado vías de control de la fuerza de trabajo creativo o ciberproletariado, que incluye tanto a los trabajadores formalmente empleados en la industria como a quienes participan de la cultura del videojuego (jugadores, modificadores, coleccionistas, archivistas, aficionados, etc.).

Además, la estructura de la industria de videojuegos contiene distintos estudios y empresas de desarrollo que varían de acuerdo al lugar que ocupan en los circuitos de producción y distribución dominantes. A comienzos del siglo XXI, diversos factores como la distribución digital, las estrategias de financiamiento alternativo ligadas al ecumenismo del capital (como el crowdfunding o los modelos free to play) y la multiplicación de los middleware, 
contribuyeron al crecimiento de la modalidad independiente de desarrollo, con marcada presencia en América Latina (Dovey, 2006; Wolf, 2008; Parker, 2013; Bidart y Uman, 2011).

No obstante, si por un lado, como señalan Cremin (2016) y Guevara (2013), los desarrolladores independientes promueven cierta tendencia hacia la innovación estética, el activismo político o el rechazo del lucro, por otro lado, también las nociones de independencia y autonomía fortalecen los intereses corporativos y debilitan a los trabajadores cognitivos precarizados, al promover una versión romántica de la cultura empresarial. Por ello, como sugieren Martin y Deuze (2009), conviene suponer que la escena indie, en lugar de oponerse, se superpone a los desarrollos corporativos, respondiendo a axiomas de mercados globales que requieren métodos de producción flexibles, así como una permanente innovación (Deleuze, 1996). No obstante, como veremos hacia el final del presente artículo, en algunos casos las exploraciones derivadas del desarrollo independiente también pueden presentarse como experimentaciones que resquebrajan las dinámicas de estriación de la industria cultural de videojuegos.

Asimismo, la escena indie también se caracteriza por su tendencia a congregarse en relación a eventos y premiaciones con fuertes características del ámbito artístico y cultural. Así, durante todo el primer lustro del siglo XXI, nacen las jornadas de desarrollo calificadas como jams que, según Sampugnaro (2014) y Turner et al. (2013), ayudan a afirmar a las comunidades de jugadores y desarrolladores en un ethos del trabajo creativo y cooperativo. Si bien los eventos estilo jam son cada vez más frecuentes, desde 2009, el crecimiento sostenido ${ }^{4}$ de la GGJ la convierte en la jornada de mayor dimensión e importancia. El objetivo de la GGJ es la invención, en un período de 48 horas, de un videojuego realizado por un grupo de participantes formado ad hoc y a partir de una serie de consignas (Guevara, 2013; Kaitila, 2012; Scott y Ghinea, 2013; Kultima y Alha, 2011). Pero, como señalan Musil et al. (2010), desde que la distribución digital intensificó los requerimientos de productos innovadores, las jams también se han convertido en formas viables de buscar una rápida experiencia de prototipado, así como de adopción de las dinámicas productivas del capitalismo cognitivo.

De acuerdo con el periodismo especializado y con los grupos de estudio de las GGJ, desde la primera edición internacional (2009) la participación de países latinoamericanos ha ido en aumento, tanto en cantidad

\footnotetext{
${ }^{4}$ Entre 2009 y 2016, la cifra de participantes en las GGJ pasó de 1650 a más de 30 000; el número de juegos pasó de 370 a más de 7200 y las sedes pasaron de 53 en 23 países, a 630 en 92 países.
} 
de concurrentes como de sedes. Según las estadísticas oficiales, entre los países latinoamericanos que participaron en la GGJ 2016, Argentina ocupa el tercer lugar en la producción, siendo el decimoquinto país a nivel mundial (detrás de Brasil y México) (Cris, 2016). Dicha edición, celebrada en Argentina, contó con 13 sedes en las que se produjeron más de 130 juegos.

\section{Metodología}

El estudio aquí reseñado se inscribe en la tradición de investigación cualitativa, según los supuestos ontológicos de las teorías que utilizamos, y está en resonancia con los fundamentos epistemológicos del paradigma constructivista (Vasilachis, 2007; Denzin y Lincoln, 2005). Contó con un trabajo de campo ${ }^{5}$ desarrollado durante tres ediciones de las GGJ (2014, 2015 y 2016) en la sede ${ }^{6}$ de Santa Fe capital (Argentina). Para la recopilación de información se utilizó la técnica de la observación participante (Hammersley y Atkinson, 1994; Scribano, 2008), combinada con diversas entrevistas en campo que versaron sobre las modalidades de producción y las perspectivas de los participantes. Por razones éticas nos referiremos a los grupos con los nombres de sus juegos alterados y para los integrantes utilizaremos iniciales ficticias. Nombraremos específicamente tres grupos en los cuales participamos: "Blanco y Negro" (2014), "Juan, el corredor" (2015) y "Ritual” (2016). Al mismo tiempo, quisiéramos señalar que en estas instancias de recopilación de información no ocultamos nuestra condición de investigadores y que, gracias a las entrevistas y discusiones sostenidas en estas jornadas, pudimos atisbar los pasos en los que la formación del cognitariado es acompañada, como veremos más adelante, por vectores de experimentación que pueden ser presentados como líneas de fuga.

Por otra parte, como señalan Kultima y Alha (2011), Locke et al. (2015), Pirker y Voll (2015) o Fowler et al. (2013), así como los mismos participantes de las GGJ, muchos de los procesos señalados como partes esenciales del desarrollo profesionalizado de videojuegos están condensados en las 48 horas seguidas de invención y en ellas se presentan las condiciones de trabajo del cognitariado en formación. Además, estos eventos permiten contemplar cómo funciona el agenciamiento de videojuegos en tanto

\footnotetext{
${ }^{5}$ Además del trabajo de observación participante, nuestra investigación incluyó dos instancias importantes de recopilación de información que, por cuestiones de espacio, no podemos reseñar aquí. En primer lugar, una reconstrucción genealógica de las racionalizaciones de las prácticas lúdicas y, en segundo lugar, un conjunto de análisis ludológicos comparados de cincuenta videojuegos desarrollados en las GGJ (de la citada sede), que nos permitió abordar en profundidad la dinámica de las reterritorializaciones artificiales del videojuego.

${ }^{6}$ Esta sede fue organizada en y por una universidad nacional perteneciente al sistema de educación superior público y gratuito de Argentina.
} 
motor de la articulación entre sujeción social y esclavitud maquínica del cognitariado, permitiendo recabar empíricamente las vías a través de las cuales las industrias culturales del juego se constituyen en un complejo reservorio de la fuerza de trabajo contemporánea.

\section{Sujeción de desarrolladores y jugadores: la empresa tiene un alma} Según Lazzarato (2014) y Berardi (2009), la axiomática capitalista pone en circulación modelos de subjetivación a través de modos de semiotización significantes o molares que, siguiendo a Foucault, encuentran en la promoción del emprendedor la figura de la sujeción propia de la gubernamentalidad neoliberal. La ubicuidad de la figura del empresario de sí se manifiesta en el impulso de transformar a cada individuo en un negocio y al conjunto del cuerpo social en un mercado competitivo de empresas, al tiempo que supone una forma de producirse a sí mismo que exacerba la autoexplotación y la autodominación. Como resume Rowan: "Uno puede ser el máximo accionista de sí mismo, pero también su máximo explotador" (2010: 69). En este punto, la figura del asalariado, paradigmática de la sujeción, comienza a mutar hacia el régimen económico de la esclavitud maquínica y supone la triste novedad que Deleuze (1991) señalaba: "Se nos enseña que las empresas tienen un alma, lo cual es sin duda la noticia más terrorífica del mundo". ${ }^{7}$

Así, no resulta extraño que durante el transcurso de nuestras observaciones en las GGJ hayamos presenciado la formación de dos microemprendimientos de desarrollo de videojuegos. Algunos integrantes del grupo cuyo trabajo observamos durante 2014 ("Blanco y Negro"), formaron un estudio de cuatro trabajadores que deseaban llevar a cabo una experiencia "sin jefes" y con proyectos propios. Una autonomía que para Rowan (2010) forma parte de los dispositivos subjetivos de la gubernamentalidad contemporánea, y que Míguez y Zangaro (2013) interpretan como un motivo fundamental de la alta rotación de trabajadores del sector. Según comentaba "L", la principal producción del estudio en ciernes eran juegos casuales y el canal de distribución era Google Play, que permitía monetizarlos. En esa ocasión, pudimos notar la disconformidad del desarrollador con la vorágine de la producción para una online store que obtura las exploraciones de mecánicas y estéticas. Esto pone de manifiesto el tipo de estriación sobre el agenciamiento de videojuegos en el mercado global (Cremin, 2016; Pedercini, 2014). Pero no se trata de un caso aislado, también en el grupo "Juan, el corredor" varios de sus integrantes estaban conformando un microemprendimiento

\footnotetext{
${ }_{7}$ Aquí hemos optado por la traducción más fiel al original, que se encuentra en Deleuze (1991).
} 
sostenido en sus producciones de las GGJ, experiencia replicada también en otros equipos de desarrollo. Además, varios de los participantes de las jams pertenecían a estudios de desarrollo ya establecidos en Santa Fe. Esto viene a confirmar tanto la tendencia a la profesionalización de los desarrolladores, como la conexión entre estas jornadas y las vías de reclutamiento; aunque también evidencia que estas jornadas son vividas como espacios liberadores que permiten poner en cuestión las estratificaciones de la industria a través de la apertura de zonas de experimentación. Estas características se exacerban cuando las mismas tienen lugar en espacios académicos que incluyen dimensiones reflexivas, como es el caso de la sede en la que llevamos a cabo nuestra observación.

Si volvemos a la potencia de las jams para generar compañías startups a partir de la publicación de sus creaciones, este aspecto no ha pasado desapercibido para autores como Kjæhr et al. (2015) que buscan métodos para "reconvertir las energías" de los eventos en esfuerzos empresariales, dado que estos microemprendimientos constituyen el centro del mito heroico de la industria de videojuegos, al tiempo que son el reverso de la precariedad y de la flexibilidad laboral.

Hay que tener en cuenta que estamos describiendo puntos de subjetivación que empujan al cognitariado como vectores de fuerza hacia una sujeción y no queremos decir, en absoluto, que los trabajadores cognitivos reproduzcan conscientemente las bases del neoliberalismo. Esta forma de sujeción o racionalidad de las prácticas está latente en todas nuestras observaciones de las GGJ, pues, como señala Guevara (2013), hay dos discursos dominantes en los desarrolladores independientes: uno tiende hacia las cualidades artísticas del estilo y otro hacia la cultura del emprendimiento.

Otra dimensión estrechamente ligada a la sujeción es la posibilidad de ver las jams como superficies donde se asientan modalidades de formación permanente. En el marco de un capitalismo que se apoya en la producción social de conocimiento, Boutang (2011) y Paltrinieri (2013) señalan que la empresa se motoriza con un stock de competencias (o capital humano) definidas por los réditos a futuro. Por ello, quizás, el tema transversal de los investigadores especializados es el de las comunidades de prácticas y aprendizajes ligadas directamente a las habilidades claves de desarrollo (Scott y Ghinea, 2013; Arya et al., 2013; Fowler et al., 2013; Kultima y Alha, 2011). Pero también la formación permanente es un resultado de la división de tareas en el trabajo cognitivo, ya que, según Míguez (2013), a diferencia del capitalismo industrial, la producción cognitiva no reposa sobre una organización homogénea y estandarizada, sino en redes y complejos entramados de cooperación. De ahí también el énfasis puesto 
en el aprendizaje social en las GGJ. Se trata de prácticas vinculadas al pliegue de la sujeción social del capitalismo contemporáneo, que estructura no sólo las dinámicas cognitivas sino la volición misma, amalgamando la producción deseante y la producción social (Deleuze y Guattari, 1997).

Por ello, Sampugnaro et al. (2014) y Pirker et al. (2016) pueden sostener que las GGJ se caracterizan por ser una instancia perfecta para la formación permanente de nuevos desarrolladores y para la actualización de los que ya están trabajando en la industria. De hecho, en la mayoría de las recapitulaciones sobre la experiencia que hacen los desarrolladores al final de las GGJ, una de las regularidades más importantes es el valor asignado a las mismas como instancias de aprendizaje. Un caso ejemplar es el de un estudiante de ingeniería en sistemas ("M") que durante el postmortem ${ }^{8}$ confiesa: "me gusta jugar pero nunca había estado metido en nada de esto [el desarrollo] y, la verdad, pocas veces se aprende tanto en tan poco tiempo". Sin embargo, no se trata de un caso aislado; otros participantes, como "H" del grupo "Ritual" (2016), destacan también el aprendizaje en codificación de middleware. No obstante, como veremos hacia el final, cuando ese aprendizaje se acompaña de la apertura del corazón del funcionamiento técnico, estamos en presencia de un tipo de juego que permite augurar contrapuntos a las tendencias hacia la automatización de los procesos productivos (Simondon, 2014).

Iniciamos este apartado con la cita de Deleuze, pero hay otro sentido de esa noción de la Posdata sobre la empresa que deviene un alma, un gas. Se trata, como lo habían advertido junto con Guattari, de que el marco de valorización del capital se ha extendido a toda la sociedad (Rossi, 2018). Como ya expresamos, la definición misma del cognitariado excede la relación salarial y abarca al conjunto de las personas implicadas en el trabajo digital (digital labor). La masa de trabajo digital impago, típico en las jams, conduce a una revalorización en términos concretos, pues en las miles de líneas de código y en el permanente modelado de assets, se encuentran soluciones que pueden ser inscritas en paquetes de software de tipo propietario (Cardon y Casilli, 2015). De hecho, las jams son auspiciadas por empresas multinacionales que se convierten en potenciales contratistas de los desarrollos prototipados, pero que además forman comunidades de potenciales clientes y usuarios; por ello, los documentos

\footnotetext{
${ }^{8}$ En la industria de videojuegos, este término suele referir a recapitulaciones de los problemas y obstáculos que se presentaron durante el desarrollo de cada producción, así como a las estrategias que se adoptaron para sortearlos. En el contexto de las GGJ, esta práctica narrativa tiene lugar al final del evento en el marco de las presentaciones de diseñadores, programadores y gestores abocados a los distintos proyectos.
} 
oficiales de la organización de la GGJ dirigidos a posibles patrocinadores subrayan haber logrado gestar la comunidad de desarrolladores más grande y "dirigir la creatividad global". Eberhardt (2016), reflexionando sobre la evolución de las GGJ, ha sugerido que las intenciones comerciales de las jams no aparecen de forma completamente transparente ante la comunidad de desarrolladores. De hecho, tanto valor tienen para los patrocinadores estas comunidades que el rango más alto se queda con el beneficio de dirigirse directamente a la comunidad. En un mundo del control donde, tal como lo supone Deleuze en su Posdata, la cifra y las bases de datos suplantan a las masas y al individuo gestando lo dividual, no es extraño encontrarse con este tipo de comercialización que se funda en el Big Data (gubernamentalidad algorítmica o modulación).

Como era de esperar esos patrocinadores son, en su mayoría, empresas multinacionales que tienden a crear fidelidad de sus comunidades. Entre ellas no es extraño encontrar compañías de plataformas de desarrollo colaborativo especializadas en manufactura de software, modelación e inteligencia artificial; pero también se destacan firmas que producen sistemas operativos y buscan incrementar tanto su caudal de juegos como de aplicaciones para revitalizar sus productos, así como publishers que posibilitan tal revitalización y middleware de desarrollo (kits de creación, game engine, etc.). Su valor proviene del cúmulo de contenidos digitales concentrados en las comunidades del cognitariado.

Como dijimos, estas compañías interpelan al cognitariado bajo la fórmula de la sujeción usuario-productor con asiento en la formación de comunidades. De ahí que se observe aquí lo que Berardi (2009), Boutang (2011) o Dantas (2012) subrayaron sobre un capitalismo cognitivo que, apropiándose de la fuerza afectiva y de comunicación de los trabajadores, también subsume, en parte, su capacidad de formar comunidades y la pliega como una forma de generar un volumen de innovaciones crecientes. Por ello Citton (2016), al evaluar esta captura rentística por parte de las empresas de radicación californiana (pero también asiática), sostiene que además de asegurarse una ganancia anclada en la extracción global de riquezas, estas plataformas oligopólicas dirigen nuestra imaginación en modos de subjetivación del control (asservissement machinique).

\section{El hombre del control es ondulatorio}

En diversos análisis de las sociedades de control, uno de los puntos singulares es la creación de un continuo de trabajo, sobretrabajo, diversiones, descanso y ocio que redunda en formas de explotación diferentes a las descritas para el trabajo industrial. Estas modalidades se arraigan en los 
procesos que Deleuze y Guattari identificaban como esclavitud maquínica generalizada o extensión de la valorización del capital a todo el cuerpo social por los agenciamientos massmediáticos -entre los cuales Guattari (2009) considera al juego. Por ello, no es extraño que para Boutang (2011) los videojuegos apareciesen como una forma de disciplinar la atención, o que para Dyer y De Peuter (2009) configurasen una vía en la que el CMI excita, moviliza, entrena y explota al cognitariado, reincorporando el juego al trabajo cognitivo. Evidentemente, las GGJ son un ejemplo característico de este fenómeno.

Teniendo en cuenta estas transformaciones, algunos autores como Kücklich utilizan el neologismo playbor y otros como Rugill se referirán a game-work, híbridos que denotan la alianza entre la excitación del juego y un proceso impago de trabajo. Estos procesos son centrales, como sostiene Fuchs (2014), en las industrias culturales massmediáticas que se basan en los contenidos generados por usuarios y que se apoyan en audiencias participativas. Por lo tanto, al lado de los juegos originales generados por desarrolladores independientes, hay que colocar otra de las vías más frecuentadas por este trabajo digital como las modificaciones sobre juegos ya existentes. Si bien, para algunos autores, como Galloway (2006), el modding permite señalar algunas vías de resistencias, para otros no ha dejado de ser alentado por los mismos publishers como una subcontratación de la innovación de los consumidores para lidiar con el riesgo en los mercados globales (Kerr, 2006). Las jams son la expresión más profunda de la cultura de las modificaciones sobre juegos existentes, tanto en el ámbito de las mecánicas como en el de las temáticas y del universo de imágenes (audiovisuales-táctiles) relacionadas con estas. En nuestras observaciones hemos notado sistemáticamente que todos los grupos estructuraban sus trabajos a partir de vastas ludotecas, que proveen dinámicas, reglas y estructuras de juego o formas de expresión del agenciamiento de videojuegos que fundan la generación de atención o engagement (Citton, 2016).

Pero también el desdibujamiento entre juego y trabajo cognitivo es rastreado por diversos autores en los comienzos mismos de las industrias de Silicon Valley. Para Fuchs (2014: 227) se trata de un "espacio social en el que el trabajo es juego y diversión”, y es correlativo a la presentación del trabajo como más libre, cuando es más flexible y precario. Asimismo, de acuerdo con Kerr (2006), la cultura del trabajo en los estudios es proclive a exacerbar características como ambientes distendidos en los que se favorece el lazo social a través de actividades lúdicas, regímenes alimenticios y comodidades que permiten jornadas de trabajo de 12 a 14 horas, así como una gran implicación basada en proyectos creativos. En lugar de ser una ética del trabajo, los estudios empíricos en estas industrias subrayan una ética del 
juego cuyo punto culminante son las jams. En esa misma línea, Locke et al. (2015) y Grace (2016) pueden encontrar el principal atributo de las jams en su espíritu juguetón o jocoso (playful), que les permite presentarlas como una experiencia o estado de juego. También es visible este elemento en la valoración que la comunidad de desarrolladores hace de las GGJ.

Nosotros consideramos que en el caso de la analogía de los autores, en realidad se trata de una demostración de la estratificación del agenciamiento de videojuegos como actividad productiva; una captura de su poderosa fuerza para abrir espacios de socialización. Esta cualidad analizada por E. Goffman (1972) permite suponer que las GGJ, a menudo vistas como reuniones focalizadas de desarrolladores, también pueden ser contempladas como espacios de formación de grupos de desarrolladores a partir de jugadores. Pero también Goffman habilita pensar los dos sentidos paralelos en los que puede entenderse el agenciamiento de videojuegos desde Deleuze y Guattari: por un lado, en tanto potencia para abrir espacios de socialización (como gaming encounters) y, por otro, montado sobre el engagement del acto de jugar un juego (game playing). Ambas son dimensiones sociales y culturales que se apoyan mutuamente como dos engranajes de una compleja operación de individuación y permiten trabajar la relación entre sujeción y esclavitud maquínica; pues, aunque aquí no tengamos espacio para su discusión en términos de la semiótica deleuzoguattariana, el engagement forja reterritorializaciones artificiales, ritornelos o agenciamientos maquínicos de cuerpos que aseguran la circuitería de los sistemas hombres-máquinas de comunicación e información necesaria para el asservissement machinique (cfr. Rossi, 2018).

Avanzando en nuestro argumento, señalemos que la estructura espacial de las GGJ, como la de los estudios que analiza Dovey (2006), surge de las disposiciones de encuentros de juego (gaming encounters). Se trata de espacios abiertos y joviales; por ello, diversos autores han señalado que las producciones independientes de videojuegos están impregnadas por un carácter humorístico e irónico. De hecho, en todas las GGJ y en particular en la del 2016, hemos visto que a menudo la estructuración misma de las ideas estaba pensada a partir de humoradas en el interior del grupo. En algún punto, como han supuesto Míguez y Zangaro (2013), este espíritu jovial permite encontrar en el trabajo la clave de la realización personal. No obstante, los sociólogos advierten que nunca se trata de un puro juego. Evidentemente el problema es mucho más complejo, puesto que las GGJ, como las dinámicas flexibles de los estudios, se apoderan de la capacidad de gestar encuentros de juego. En otros términos, el dispositivo de trabajo cognitivo se monta sobre o afecta (en tanto que el poder es una afección del deseo) a las componendas de juego. 
Por otra parte, las 48 horas continuas de desarrollo de videojuegos permiten ver lo que habitualmente la sociología del trabajo describe como confusión de los tiempos productivos y horarios flexibles. Para Berardi, la noción de productividad misma se vuelve difícil de definir cuando la relación entre tiempo y cantidad del valor producido no es clara. Por ello, no es extraño que durante todas las jornadas hayamos registrado momentos en que los desarrolladores, para romper con la monotonía del trabajo o como forma de esparcimiento, se disponen a jugar juegos multijugador. Asimismo, vinculado a la fusión del tiempo de juego y el tiempo de trabajo, hay que señalar que en los tres años pudimos observar fenómenos muy particulares de excitación y, en cierta medida, quiebre del ritmo circadiano. Ante nuestras preguntas, uno de los integrantes del grupo "Blanco y Negro" equipara directamente la excitación del desarrollo con la del juego: "programar videojuegos es tan excitante como jugarlos". Esta declaración confirma en cierta medida la identidad gamer que Gómez (2014) identifica como parte de la biopolítica contemporánea y que para nosotros no es sino parte del asservissement machinique. También en los grupos del 2015 y 2016, la invención de los juegos afecta profundamente los ritmos de sus integrantes.

La afectación de los tiempos y regímenes alimenticios al servicio del apretado esquema de desarrollo, compromete a quienes deciden continuar diseñando durante las dos jornadas de forma continua, ${ }^{9}$ sin descanso. Como sugiere Berardi (2009), el cognitariado es expuesto a una activación semiótica de su sistema nervioso, una electrocución afectocognitiva que nace del filum maquínico y de reterritorializaciones por medios artificiales (Guattari, 2009; Rossi, 2018). De hecho, en la mayoría de los grupos observados sus integrantes han decidido no dormir, pero cuando la aceleración cede lo que surge es una fatiga intensa y un abatimiento que pone de manifiesto otra experiencia propia de estas jornadas: dormir junto a la estación de trabajo (Montes Cató, 2010). Este fenómeno, festejado por autores como Turner et al. (2013), se debe relacionar con la fatiga propia del trabajo en la pantalla (Boutang, 2011), aunque quizás el dato más interesante es que estas prácticas son desalentadas por los organizadores locales de la GGJ.

Para nosotros, esta estructuración inmanente de los ritmos de actividad está codificada por una de las características más singulares del desarrollo de videojuegos: el diseño iterativo (Flanagan, 2009). Se trata de un método en el cual las decisiones son tomadas en base a las experiencias o

\footnotetext{
9 En la presentación grupal del 2014, uno de los integrantes de un grupo en el cual no seguimos la observación participante declaró: "la experiencia de estar así, sin tiempo, sin poder dormir, es genial...”.
} 
gameplay suscitadas por el prototipo que es jugado, evaluado, ajustado y jugado nuevamente. Esta circularidad es un factor decisivo para fusionar trabajo y juego, como sugiere uno de los manuales de desarrollo más citado: "[a] través del proceso de diseño iterativo, el diseñador se vuelve un jugador y el acto de jugar se vuelve un acto de diseño" (Salen y Zimmerman, 2004: 12). Así, todo el proceso de diseño y desarrollo de los videojuegos en las GGJ está estructurado a partir de una iteración constante de las versiones y un testeo interminable de los prototipos y mecánicas, que puede colocarse en la base misma de la esclavitud maquínica como sistema de comunicación e información (o ritornelo). Por supuesto, esta cualidad de la iteración depende también del propio agenciamiento de videojuegos, específicamente como playing a game.

Como señala Fuchs (2014), estas mismas condiciones de juego-trabajo se pueden convertir en situaciones de explotación. Ahora bien, tanto la categoría de game-work, como la de playbor (unidas a menudo a la noción de prosumidor) no iluminan exactamente lo que intentamos estudiar, pues nuestro interés fundamental aquí es ver cómo el agenciamiento de videojuegos expresa el diagrama de poder de la sociedad contemporánea, exacerbando la molecularización del control para mostrar su rostro más infame: su productividad o el hecho de poder controlar los acontecimientos principales de una multiplicidad numerosa en un espacio abierto (Deleuze, 2014). Todos estos ciclos iterativos que van desde la indistinción con el trabajo hasta los ritmos alterados de producción y excitaciones de tareas constantes en los "controlatorios", confirman aquello que Deleuze señalaba al decir que "[e]l hombre de las disciplinas era un productor discontinuo de energía, pero el hombre del control es más bien ondulatorio, en órbita sobre un haz continuo. Por todas partes, el surf ha reemplazado a los viejos deportes" (Deleuze, 1996: 283). Esta metáfora del surf y la producción continua y ondulante de energía, habla más del modo de semiotización de la esclavitud maquínica que de la sujeción social, la forma de axiomatización del capitalismo contemporáneo.

\section{Del trabajo por proyectos (deadlines y prototipos)}

En los tres grupos que hemos observado durante las GGJ, registramos una profunda división de tareas que se sostenía en las competencias y habilidades de la organización del proceso de producción de videojuegos. De acuerdo a Buttfield-Addison et al. (2016), que estudian grupos de desarrollo en las GGJ, esta división entre programadores, game designers y artistas se basa en que el agenciamiento de videojuegos es inseparable de un modelo de diseño compuesto por mecánicas, dinámicas y estéticas entrelazadas (MDA, 
por sus siglas en inglés). Así, la división de tareas se efectúa a partir de una comprensión consciente del proceso de diseño del juego, que en las GGJ se especifica en los postmortems de los grupos. Pero también este modelo está ligado a la diversidad profesional del cognitariado que es atraído hacia el desarrollo de videojuegos. Por ejemplo, el grupo del 2016 estaba constituido por estudiantes de arquitectura, diseño gráfico, mecatrónica, ingeniería en sistemas, programación, etc. Esta diversidad se repite en la mayoría de los grupos de las GGJ.

Por otra parte, el modelo MDA es parte del conocimiento tácito de los jammers y en los tres años que hemos observado se repetía la división de tareas de acuerdo al mismo, formando prácticas y rutinas de trabajo naturalizadas que ponen en primer plano los saberes entrelazados. Según Míguez (2013), esta división cognitiva del trabajo se basa en el fraccionamiento del proceso de producción de acuerdo a la naturaleza de los conocimientos movilizados por cada proyecto. Se trata de un trabajo relacional y reticular, un proceso de valorización sobre signos ricos en conocimiento específico y cada vez menos intercambiable (Berardi, 2009); por lo tanto, también es más personalizado y recombinante en redes digitales donde la función de comando no está jerárquicamente impuesta, sino que se desterritorializa permeando cada fragmento del tiempo de trabajo. En la esclavitud maquínica el control es incorporado al flujo semiótico. Esta dependencia de la fluidez automática de la red, se pone de manifiesto en un sinfín de operaciones que hemos observado durante todas las GGJ (impensables sin Internet y sin la tecnología celular-conectiva).

Además de la división de tareas, también son características las descripciones del proceso de invención subdividido en distintas etapas, donde las GGJ son jornadas en las que se vuelven ejemplares las instancias de preproducción y producción. En estas etapas se condensan los mayores esfuerzos creativos ligados al trabajo por proyecto y a la realización de prototipos. Como señalan Musil et al. (2010), las jams son una de las formas más efectivas para prototipar de modo abreviado videojuegos que luego podrán ser o no puestos en el mercado. Por ello no es extraño, como sugieren Kjæhr et al. (2015), que desde las compañías de desarrollo profesionales se contemplen estas jornadas como ideales para crear ambientes creativos de trabajo. Dado que, aun cuando no se cuenta con rutinas o esquemas de producción específicos, el armazón (framework) de las jams se vuelve un componente central para aumentar la productividad. Lograr el prototipado en 48 horas es una forma de emular las deadlines y proveer, como sugieren Fowler et al. (2013), un campo de extrema tensión que no deja de afectar los ciclos iterativos del diseño. 
Así, durante todas las GGJ hemos registrado momentos en los que la misma comunidad de desarrolladores insiste en la brevedad de los proyectos de acuerdo a las fechas límites. A su vez, estas limitaciones temporales modulan el devaneo de las ideas e imágenes que crecen en los grupos, pues el límite de las 48 horas de las GGJ, como vía de sujeción, jamás se desdibuja en la conciencia de los participantes y, como pudimos observar en el grupo del 2016, siempre está asociado al cumplimiento de la consigna. Incluso si es laxo, este límite no deja de permear la voluntad; de hecho, uno de los fenómenos más extraños, para el observador que no pertenece a estas comunidades, es el trabajo más allá del tiempo específico del evento. Los tres grupos que hemos acompañado en su producción, una vez concluida la jam dedicaron varias horas extras para terminar y subir su juego a la web.

Por lo tanto, en el marco de las interpelaciones de las jams como espacios para la creación de prototipos, hemos observado tensiones en los grupos acerca de compartir un concepto de juego (open source) o presentarlo como innovación comercial (restringiendo su circulación). De hecho, en el grupo "Juan, el corredor" algunos integrantes comenzaban a expresar reparos a la hora de subir el juego, el código y los elementos audiovisuales a la página oficial de GGJ. Para uno de los participantes ("I") el trabajo de los artistas era lo suficientemente creativo como para registrarlo bajo propiedad intelectual, al tiempo que la idea misma del juego, basada en la complementariedad de dos mecánicas (puzzle y runner), era valorada como una importante innovación comercial. En contrapartida, otros integrantes del grupo (como "M" y "J") subrayaban el ethos de una economía contributiva instando a publicar de forma libre y gratuita. En este caso en particular, el grupo, a través de una votación, publicaría de forma libre el código, el concepto y los elementos del juego. No obstante, quienes intentaban el resguardo comercial acelerarían la presentación de los obstáculos al punto de hacer imposible sortear las dificultades a través de la gameplay. Esto es una muestra de cómo el régimen de propiedad puede afectar a las mecánicas y dinámicas de los juegos, pero también pone de manifiesto las exploraciones de los participantes en los impossible games, pues incluso aquellos que querían compartir el proyecto apreciaban la experimentación con dinámicas que, al sacar al jugador del lugar de pasividad construido por la industria de videojuegos, tienen una suerte de poder desterritorializante (ver más adelante). Ciertamente, las prácticas de prototipado, como sostienen Corsín Jiménez y Estalella (2017) y Corsín Jiménez (2014), cuando logran escapar a las dinámicas de la axiomática capitalista, pueden convertirse en zonas de experimentación caracterizadas por la cualidad abierta de sus fuentes estructurales y técnicas que permiten la libre distribución, 
copia y modificación (alcanzando en ciertos momentos, como veremos más adelante, aristas políticas novedosas).

$\mathrm{Al}$ mismo tiempo, subrayemos que el lugar performativo de la propiedad intelectual no es una novedad y está estrechamente ligado al conjunto de enunciados que se refieren a la búsqueda de monetización de los desarrollos, tal como señalan autores que se apoyan en la noción de capitalismo informacional y cognitivo (Zuckerfeld, 2013; Vercellone, 2011). Además, debe recordarse que los estudios independientes y freelancers, sujetos a contrataciones, tienen en los portfolios una carta de presentación fundamental, tanto para conseguir clientes como para ser contratados (Wearn y McDonald, 2016), porque las deadlines y los prototipados corresponden a un capitalismo ya no para "la producción, sino para el producto, es decir para la venta y para el mercado" (Deleuze, 1996: 284). No obstante, esta tendencia hacia la formación de microempresas entra en disputa con las formas de compartir o las presiones de las comunidades, como señala Fuchs (2014), que bregan por una cultura del código abierto y de bienes comunes para los estudios (Guevara, 2013). Pero no se trata de una contradicción; de hecho, es característico de los procesos de implicación subjetiva que el trabajador cognitivo esté predispuesto a aceptar remuneraciones simbólicas, como se puede observar en todas las GGJ (Míguez, 2013).

Sin embargo, el aprendizaje no solo pasa ahora por el timing de los proyectos, sino también por saberes relativos a los software de desarrollo (Fowler et al., 2013). Aquí las empresas de herramientas informáticas encuentran anclaje en comunidades que, como ya dijimos, son moduladas bajo capacidades que se describen como de rápida obsolescencia y sometidas a una continua evaluación. El otro costado de esta innovación es una fuerza de trabajo que ha de acostumbrarse a la constante actualización de sus conocimientos.

Hasta mediados de la década de los noventa, el desarrollo de videojuegos en computadora debía partir de cero, codificando todos los elementos, diseñando cada imagen; pero desde mediados de la primera década del siglo XXI, el crecimiento de los estudios independientes ha sido acompañado por una ampliación de los paquetes de software de desarrollo o middleware. Algunas de estas herramientas suplantan o facilitan la codificación (Martin y Deuze, 2009). De hecho, la industria de los middleware ha crecido como oportunidad de negocios para empresas de alta complejidad tecnológica que apuntan como potenciales clientes no sólo a los estudios, sino principalmente a desarrolladores indie, hobbistas y fanáticos de videojuegos (Arya et al., 2013; Hill-Whittall, 2015). 
En los tres grupos que observamos, así como en otros de las GGJ, el game engine utilizado fue Unity. Se trata de un caso paradigmático de los middleware de sujeción usuario-productor pues, aunque cuenta con versiones libres y funcionales, está hecho para ser licenciado. Además, cuenta con una vía de distribución de los juegos creados con su motor y tiendas online especializadas en assets y scripts. El gran soporte documental de este middleware (característica ponderada por los integrantes de los grupos), se debe a la organización provechosa de una extensa comunidad de desarrolladores que discuten en los foros de la empresa sobre las posibilidades de desarrollo. Se trata de una compañía ejemplar del capitalismo cognitivo que extrae su valorización de las comunidades de clientes y de la captura de saberes sociales (Fuchs, 2014; Cardon y Casilli, 2015). Así, si estas compañías de middleware, por un lado, alientan una mayor diversidad al abrir el campo de la producción, por otro, se valorizan por el tamaño de las comunidades y desarrollos que manejan.

\section{Palabras finales (líneas de fuga)}

$\mathrm{Al}$ comenzar este artículo hemos propuesto la figura de un péndulo entre sujeción y esclavitud maquínica que coordina los procesos de formación del cognitariado. Hemos visto que esos procesos estaban ligados a la interpelación al emprendimiento, la formación permanente, la gestación de comunidades de desarrolladores-jugadores, la fusión de los tiempos y dinámicas de producción a la medida de deadlines. Ahora quisiéramos señalar dos ejes estrechamente relacionados con las prácticas que hemos observado.

En primer lugar, hay un eje teórico que hemos abordado en otros trabajos y que permite entender la complementariedad entre las mutaciones en los modos de subjetivación y en los modos de semiotización para el capitalismo contemporáneo (cfr. Rossi, 2018). En su esencia, dicho argumento sostiene que, si bien muchos de los procesos de la sujeción están vinculados a las líneas molares de los agenciamientos controlatorios y pueden ser cabalmente comprendidos en los márgenes del régimen de signos de la rostridad (o de la significancia e interpretación), la esclavitud maquínica pone en evidencia la preponderancia de nuevos modos de semiotización que se afirman en las modalidades del ritornelo. Se trata de una temporalidad específica que asegura la territorialización de los agenciamientos como punto de contacto entre semióticas de la corporeidad y semióticas asignificantes (de la información, del silicio, de la computación, etc.). Estas semióticas no se apoyan solamente en la sujeción (disciplinaria) de un sujeto individuado, sino en una realidad dividual y tienen como asiento principal los pequeños ritmos 
sociales (ritornelos) abiertos por los agenciamientos de las formaciones sociales del control (como los videojuegos).

En segundo lugar, existe un eje estrictamente ligado al anterior, pues para Deleuze y Guattari la noción de dividual señala la conformación de un pueblo que falta y que puede unir las artes con los hombres que luchan. Así, si por un lado las GGJ son ejemplares en la adopción de dinámicas de trabajo cognitivo, por otro lado, las comunidades de desarrolladores-jugadores suelen presentar a estos eventos, fundamentalmente, como espacios para explorar ideas creativas que van a contramano de los contenidos convencionales de la industria mainstream (Westecott, 2014). En particular, como hemos subrayado anteriormente, estas jornadas no permiten una lectura lineal y pueden servir de anclaje para zonas de experimentación que tienden a abrir a los agenciamientos de videojuegos a procesos de devenir. Como vimos en el acápite anterior, ya el hecho de compartir los enormes esfuerzos realizados en las GGJ en forma libre y gratuita señala una suerte de fuga de la dinámica de apropiación del capital (entrando sólo parcialmente en los procesos de sujeción) hacia economías contributivas cuyo perfil aún está en vías de formación (Stiegler, 2015). También por ello, no es extraño encontrar profundos procesos de desterritorialización en los que el asservissement machinique puede revelarse como un modo de subjetivación en tránsito hacia lo que Deleuze y Guattari llamaban era posmediática o arte del control y que se componen de devenires minoritarios. En todas nuestras observaciones hemos encontrado líneas de fuga ejemplificadas por la apropiación de grandes ludotecas y, a raíz de que estas fueron realizadas en una universidad pública, hemos encontrado momentos en los que se animaba a quebrar las estriaciones molares de la industria. Se trata de una suerte de devenir-niño soportado por el juego que, extrayendo bloques de infancia que no se reducen a una infantilización (Deleuze, 2006), permiten arrancar de las semióticas moleculares de los videojuegos procesos que van a contramano del mainstream. Encontramos así verdaderas intenciones estéticas y políticas detrás de algunos desarrollos que, en primer lugar, ponen en cuestión el plano representacional (molar) de la industria de videojuegos. Este cuestionamiento pasa por el tratamiento de problemas ecológicos, la deconstrucción de retóricas violentas o, como registramos en las discusiones de los integrantes de "Juan, el corredor", problematizaciones de la identidad de género, así como del lugar de la mujer (en la industria y en los contenidos de sus juegos).

En segundo lugar, al mismo tiempo hay desarrollos que urden la trama estética y liberan lo que podríamos llamar líneas moleculares de los videojuegos. En especial, esto se cifra en la aparición de tendencias de game art, así como 
en la construcción de juegos que desafían al jugador a salirse de las mecánicas y dinámicas pasatistas universalizadas con los social games (Pedercini, 2014). Parte del juego del devenir-niño de lo mayoritario consiste en extraer esos bloques de infancia que son verdaderos fragmentos de experimentación.

Concomitantemente, contra las estrategias de sujeción ligadas a la opacidad de la masificación de la computación (Citton, 2016), muchos de los jugadores-desarrolladores toman las GGJ (en nuestro caso, alentados por los organizadores) como una vía de exploración de game engines de código abierto, así como de plataformas alternativas, y como un motor para el aprendizaje de codificación que configuran epifanías de las técnicas (tecnofanías) en el sentido de G. Simondon (2014) (o devenir-electrónica del cognitariado [Deleuze y Guattari, 1997]). Así, en las GGJ hemos encontrado participantes que se embarcaban en la exploración técnica de la construcción de hardware y software, abriendo procesos de un jugar tecnológico que se apropia de saberes y prácticas desalentados por una industria que apuesta a la progresiva automatización de los procesos productivos y a la obsolescencia programada. Con esto, los participantes ensayan lo que podría ser pensado como nuevas relaciones sociales construidas sobre procesos de individuación psíquica y colectiva, que se apoyan en realidades técnicas (o transindividuación) y que se caracterizan por poner entre paréntesis relaciones sociales (interindividuales) del consumo, la propiedad o el trabajo (subrayando la potencialidad del prototipado [Corsín, 2014], así como la posibilidad de recuperar saberes que, en términos stieglerianos, exceden a la economía del consumo).

Se trata, como considera Deleuze (2017), de una suerte de proposiciones indecidibles del cognitariado que son irreductibles a la dinámica de la axiomática capitalista, en tanto les permiten explorar agenciamientos que tienen la potencialidad para hacer fugar líneas de las formaciones sociales del control (Guattari, 2017). Por ello, en los grupos de las GGJ puede encontrarse aquello que Guattari subrayaba al decir que las máquinas técnicas tienen la potencia desterritorializante de liberar a las máquinas deseantes. También, como estos autores advierten, si el capitalismo ha mutado, las estrategias de las luchas no pueden permanecer anquilosadas y las jams se presentan como una zona potencial para abrir formas de un pensar futuro. Ciertamente estos procesos y ejes, como señala Galloway (2006), podrán ser abordados en próximos trabajos como formas de resistencias y devenires del cognitariado en formación.

Revisado por José Morales 


\section{Referencias bibliográficas}

Arya, Ali; Chastine, Jeff; Preston, Jon; Fowler, Allan (2013), "An International Study on Learning and Process Choices in the Global Game Jam”, International Journal of Game-Based Learning (IJGBL), 3(4), 27-46.

Berardi, Franco (2009), The Soul at Work. Los Angeles: Semiotext(e).

Bidart, Silvia; Uman, Verónica (2011), "La industria de desarrollo de videojuegos: Argentina”, in VV. AA. (comps.), Guerra y paz en el mundo virtual. Chile: Centro de Estudios Universitarios, 41-66.

Boutang, Yann Moulier (2011), Cognitive Capitalism. Cambridge: Polity Press.

Buttfield-Addison, Paris; Manning, Jon; Nugent, Tim (2016), "A Better Recipe for Game Jams: Using the Mechanics Dynamics Aesthetics Framework for Planning", Proceedings of the International Conference on Game Jams, 30-33.

Cardon, Dominique; Casilli, Antonio (2015), Qu'est-ce que le Digital Labor? Bry-sur-Marne: INA.

Citton, Yves (2016), "Subjectivations computationnelles à l’ère numérique", Multitudes, 62, 48-66.

Corsín Jiménez, Alberto (2014), "Introduction: The Prototype: More than Many and Less than One", Journal of Cultural Economy, 7(4), 381-398.

Corsín Jiménez, Alberto; Estalella, Adolfo (2017), "Ethnography: A Prototype", Ethnos, 82(5), 846-866.

Cremin, Colin (2016), Exploring Videogames with Deleuze and Guattari. London: Routledge.

Cris, Cristian (2016), "Lo que nos dejó la Global Game Jam 2016". Consultado el 09.02.2016, en https://cristianelcris.wordpress.com/2016/02/09/lo-que-nosdejo-la-global-game-jam-2016/.

Dantas, Marcos (2012), Trabalho com informação. Rio de Janeiro: Centro de Filosofia e Ciências Humanas da Universidade Federal do Rio de Janeiro.

Deleuze, Gilles (1991), "Posdata sobre las sociedades de control", in Christian Ferrer (comp.), El lenguaje literario. Montevideo: Ed. Nordan. Consultado el 06.02.2015, en http://www.catedras.fsoc.uba.ar/heler/deleuze2005.htm.

Deleuze, Gilles (1996), Conversaciones. Valencia: Pre-textos. Traducción de José Pérez Vázquez y Umbelina Larraceta.

Deleuze, Gilles (2006), Derrames I. Buenos Aires: Cactus. Traducción de Equipo editorial Cactus.

Deleuze, Gilles (2014), El poder. Buenos Aires: Cactus. Traducción de Pablo Ires.

Deleuze, Gilles (2017), Derrames II. Buenos Aires: Cactus. Traducción de Pablo Ires y Sebastián Puente.

Deleuze, Gilles; Guattari, Félix (1997), Mil Mesetas. Valencia: Pre-textos. Traducción de José Pérez Vázquez y Umbelina Larraceta.

Denzin, Norman; Lincoln, Yvonna (2005), The Sage Handbook of Qualitative Research. New York: Sage Publications. 
Dovey, Jon; Kennedy, Helen (2006), Games Cultures. Glasgow: Open University Press. Dyer, Nick; De Peuter, Greg (2009), Games of Empire. Minneapolis: University of Minnesota Press.

Dyer, Nick (2015), Cyber-Proletariat. Canada: Pluto Press.

Eberhardt, Richard (2016), "No One Way to Jam: Game Jams for Creativity, Learning, Entertainment, and Research", Proceedings of the International Conference on Game Jams, 34-37.

Flanagan, Mary (2009), Critical Play. New York: MIT Press.

Fowler, Allan; Koshmood, Foaad; Arya, Ali; Gorm, Lai (2013), “The Global Game Jam for Teaching and Learning”. Comunicación presentada en la 4th Annual Conference of Computing and Information Technology Research and Education, 6 al 9 de Octubre, Hamilton, New Zealand. Consultado el 10.07.2015, en http://www.citrenz. ac.nz/conferences/2013/pdf/2013CITRENZ_1_Fowler01-GlobalGameJam_v2.pdf.

Fuchs, Christian (2014), Digital Labour and Karl Marx. London: Routledge.

Galloway, Alexander R. (2006), Gaming: Essays on Algorithmic Culture. Minneapolis: University of Minnesota Press.

Goffman, Erving (1972), Encounters. Great Britain: The Penguin Press.

Gómez, Sebastián (2014), "Ludocapitalismo”, in Iván Torres (comp.), Actas de la primera Jornada transdisciplinar de estudios en gubernamentalidad. Chile: Escaparate, 315-323.

Grace, Lindsay (2016), "Deciphering Hackathons and Game Jams through Play", in Proceedings of the International Conference on Game Jams. New York: ACM, 42-45.

Guattari, Félix (2009), Soft Subversions. Los Angeles: Semiotext(e).

Guattari, Félix (2017), La revolución molecular. Madrid: Errata Naturae.

Guevara, Orlando (2013), "Cultural Production and Politics of the Digital Games Industry”. Tesis de Doctorado en Sociología, University of Edinburg, Scotland.

Hammersley, Martyn; Atkinson, Paul (1994), Etnografía. Métodos de investigación. Barcelona: Paidós.

Hardt, Michael; Negri, Antonio (2002), Imperio. Buenos Aires: Paidós.

Hill-Whittall, Richard (2015), The Indie Game Developer Handbook. London: Focal Press.

Kaitila, Christer (2012), The Game Jam Survival Guide. Birmingham: Packt Publishing Ltd.

Kerr, Aphra (2006), The Business and Culture of Digital Games. London: Sage.

Kjæhr, Emil B.; Jensen, Jane L. Hvid; Schønau-Fog, Henrik; Reng, Lars (2015),

"From Jam to Start-up: A Framework to Support Entrepreneurship at Game Jams and Production Oriented Workshops", Foundations of Digital Games Conference. Consultado el 23.04.2016, en http://www.forskningsdatabasen.dk/en/ catalog/2265486433.

Kultima, Annakaisa; Alha, Kati (2011), "Using the VNA Ideation Game at Global Game Jam”, Proceedings of DiGRA 2011 Conference: Think Design Play, 6, 2-16.

Lazzarato, Maurizio (2014), Signs and Machines. Los Angeles: Semiotext(e). 
Locke, Ryan; Parker, Lynn; Galloway, Dayna; Sloan, Robin (2015), “The Game Jam Movement: Disruption, Performance and Artwork", in Workshop Proceedings of the 10th International Conference on the Foundations of Digital Games. Consultado el 20.07.2016, en https://rke.abertay.ac.uk/en/publications/the-game-jam-movementdisruption-performance-and-artwork.

Martin, Chase; Deuze, Mark (2009), “The Independent Production of Culture”, Games and Culture, 4(3), 276-295.

Míguez, Pablo (2013), "Del General Intellect a las tesis del 'capitalismo cognitivo"”, Bajo el Volcán, 13(21), 27-57.

Míguez, Pablo; Zangaro, Marcela (2013), “El trabajo inmaterial-cognitivo y sus consecuencias para los trabajadores: el caso paradigmático del desarrollo de videojuegos”. Comunicación presentada en el VII Congreso Latinoamericano de la ALAST, Asociación Latinoamericana de Sociología del Trabajo, Julio, São Paulo, Brasil.

Montes Cató, Juan (comp.) (2010), El trabajo en el capitalismo informacional. Benavidez: Poder y trabajo.

Musil, Juergen; Schweda, Angelika; Winkler, Dietmar; Biffl, Stefan (2010), "Synthesized Essence", ACM/IEEE 32nd International Conference on Software Engineering, 2, 183-186.

Paltrinieri, Luca (2013), “Quantifier la qualité: le 'capital humain’ entre économie, démographie et éducation”, Raisons politiques, 4(52), 89-107.

Parker, Felan (2013), "Indie Game Studies Year Eleven”, DIGRA '13 - Proceedings of the 2013 DiGRA International Conference: DeFragging Game Studies, 7. Consultado el 13.05.2015, en http://courses.bloodedbythought.org/pplay/images/d/df/Parker, Felan-Indie_Gaming.pdf.

Pedercini, Paolo (2014), "Videogames and the Spirit of Capitalism". Consultado el 18.07.2016, en http://www.molleindustria.org/blog/videogames-and-the-spiritof-capitalism/.

Pirker, Johanna; Voll, Kimberly (2015), “Group Forming Processes - Experiences and Best Practice from Different Game Jams", Workshop Proceedings of the 10th International Conference on the Foundations of Digital Games. Consultado el 08.08.2016, en http://jpirker.com/wp-content/uploads/2013/09/final.pdf.

Pirker, Johanna; Kultima, Annakaisa; Gütl, Christian (2016), “The Value of Game Prototyping Projects for Students and Industry", Proceedings of the International Conference on Game Jams, Hackathons, and Game Creation Events, 54-57. DOI: 10.1145/2897167.2897180

Rowan, Jarón (2010), Emprendizajes en cultura. Madrid: Traficantes de sueños.

Rossi, Luis Sebastián (2018), “Agenciamientos en las sociedades de control”, Cultura-Hombre-Sociedad, 28(1), 177-206.

Salen, Katie; Zimmerman, Eric (2004), Rules of Play. Cambridge: MIT Press. 
Sampugnaro, Rossana; Mica, Salvatore; Fallica, Salvatore; Bonaiuto, Ambra; Mingrino, Marta (2014), "Participation at the Global Game Jam: A Bridge between Consumer and Producer Worlds in Digital Entertainment", GAME - The Italian Journal of Game Studies, 1(3), 35-45.

Scott, Michael James; Ghinea, Gheorghita (2013), "Promoting Game Accessibility: Experiencing an Induction on Inclusive Design Practice at the Global Games Jam”, Proceedings of the Inaugural Workshop on the Global Games Jam, 17-20. Consultado el 04.05.2015, en https://arxiv.org/ftp/arxiv/papers/1305/1305.4359.pdf.

Scribano, Adrián (2008), El proceso de investigación social cualitativo. Buenos Aires: Prometeo.

Simondon, Gilbert (2014), Sur la technique. Paris: Presses universitaires de France.

Stiegler, Bernard (2015), L'emploi est mort, vive le travail!: entretien avec Ariel Kyrou. Paris: Fayard.

Turner, Jane; Owen, Cameron; Thomas, Lubi (2013), "Living the Indie Life: Mapping Creative Teams in a 48 Hour Game Jam and Playing with Data", Proceedings of The 9th Australasian Conference on Interactive Entertainment. Consultado el 19.09.2015, en http://eprints.qut.edu.au/64496/.

Vasilachis, Irene (2007), Estrategias de investigación cualitativa. Buenos Aires: Gedisa. Vercellone, Carlo (2011), Capitalismo cognitivo. Renta, saber y valor en la época posfordista. Buenos Aires: Prometeo.

Wearn, Nia; McDonald, Brian (2016), "Ethos of Location and Its Implication to the Motivators of Global Games Jam Participants", in Proceedings of the International Conference on Game Jams. San Francisco: ACM, 58-61.

Westecott, Emma (2014), "Independent Game Development as Craft”, Loading..., 7(11), 78-91.

Wolf, Mark (2008), The Video Game Explosion. Westport: Greenwood Press.

Zukerfeld, Mariano (2013), Obreros de los bits: conocimiento, trabajo y tecnologías digitales. Bernal: Universidad Nacional de Quilmes.

Recibido: 29.08.2017

Aceptación comunicada: 10.01.2019

\section{Luis Sebastián Ramón Rossi}

Centro de Investigaciones Sociales y Políticas, Facultad de Ciencias de la Educación, Universidad Nacional de Entre Ríos (CISPO-FCEdu-UNER) I Consejo Nacional de Investigaciones Científicas y Técnicas (CONICET)

Alameda de la Federación 106, Paraná, Entre Ríos, Argentina.

Contacto: luissebastianrossi@gmail.com

ORCID: https://orcid.org/0000-0003-3638-5857 
Video Games and Societies of Control: The Formation of Cognitariat in the Global Game Jams In this article, we use the Deleuzoguattarian division between social subjection (assujettissement) and machinic enslavement (asservissement machinique) as the basis for understanding the formation of the cognitariat in Global Game Jams. After contextualizing these sessions and their importance for the video game development industry, we will examine the dimensions of user-producer subjection (such as the appeal to entrepreneurship, continuing training, and formation of communities) and explore dimensions of machinic enslavement (flexible work dynamics, game and labour fusion, division of tasks and projects, etc.).

Keywords: capitalism; cognitariat; Global Game Jams; labour; social relations; video games.
Jeux vidéo et sociétés de contrôle: formation du cognitariat à la Global Game Jams

Dans cet article, nous utilisons la division deleuzeguattarienne entre assujettissement social et asservissement machinique pour comprendre la formation du cognitariat lors des journées de développement des jeux vidéo Global Game Jams. Après avoir mis en contexte ces sessions et leur importance pour l'industrie du développement de jeux vidéo, nous travaillerons, d'une part, sur les dimensions de l'assujettissement utilisateur-producteur (appel à l'esprit d'entreprise, formation continue, gestation des communautés) et, d'autre part, les dimensions de l'asservissement machinique (dynamique de travail flexible, fusion du jeu et du travail, division des tâches et des projets, etc.).

Mots-clés: capitalisme; cognitariat; Global Game Jams; jeux vidéo; relations sociales; travail. 